\title{
Biological Rhythms Workshop IA: Molecular Basis of Rhythms Generation
}

\author{
S.R. MACKEY* \\ Department of Biology, The Center for Research on Biological Clocks, \\ Texas A\&M University, College Station, Texas 77843-3258
}

\begin{abstract}
Current circadian models are based on genetic, biochemical, and structural data that, when combined, provide a comprehensive picture of the molecular basis for rhythms generation. These models describe three basic elements-input pathways, oscillator, and output pathways - to which each molecular component is assigned. The lines between these elements are often blurred because some proteins function in more than one element of the circadian system. The end result of these molecular oscillations is the same in each system (near 24-hour timing), yet the proteins involved, the interactions among those proteins, and the regulatory feedback loops differ. Here, the current models for the molecular basis for rhythms generation are described for the prokaryotic cyanobacterium Synechococcus elongatus as well as the eukaryotic systems Neurospora crassa, Drosophila melanogaster, Arabidopsis thaliana, and mammals (particularly rodents).
\end{abstract}

\section{INTRODUCTION}

Internally generated, near 24-hour rhythms of behavior are observed in numerous organisms ranging from prokaryotic cyanobacteria to mammals. Driving the overt 24-hour rhythmic behaviors are oscillations that occur at the molecular level. The molecular components can be assigned to one of three basic elements within the biological clock: input pathways that relay synchronizing environmental cues to the central oscillator, the oscillator that maintains internal time, or output pathways that transduce temporal information from the oscillator to clock-controlled processes. However, as more is discovered about the complexity of the various circadian systems, the distinctions among these elements become blurred because one protein may act in more than one element of the system. In this section, the similarities and differences of the molecular components among five different circadian systems-Synechococcus elongatus, Neurospora crassa, Drosophila melanogaster, Arabidopsis thaliana, and mammals (rodents) - are described and compared.

\section{PROKARYOTIC CIRCADIAN CLOCK SYSTEMS}

Until the 1980s, circadian clocks were believed to exist only in eukaryotes. Prokaryotes, with life spans much shorter than that of a full circadian cycle, were thought to have no need to maintain a 24-hour timing mechanism. However, investigation into the existence of two incompatible processes - oxygenic photosynthesis and oxygensensitive nitrogen fixation-that occur in some unicellular cyanobacteria led to the discovery that these processes are separated temporally by an endogenous timekeeping mechanism, with photosynthesis occurring during the day and nitrogen fixation at night (Mitsui et al.

*Present Address: Department of Biology, St. Ambrose University, Davenport, Iowa 52803
1986). Further studies have shown that the circadian clock in cyanobacteria regulates the timing of amino acid uptake (Chen et al. 1991), global gene expression (Liu et al. 1995), chromosome condensation (Smith and Williams 2006), and cell division (Sweeney and Borgese 1989; Mori and Johnson 2000).

\section{Synechococcus elongatus}

Oscillator. The cyanobacterial central oscillator is composed of the KaiA, KaiB, and KaiC proteins. Accumulation of kaiA (expressed from its own promoter) and $k a i B C$ (expressed as a dicistronic message from one promoter upstream of $k a i B$ ) mRNA occurs in a circadian manner with peak expression 12 hours after entering constant light conditions (Fig. 1) (Ishiura et al. 1998). Maximum levels of fluctuating $\mathrm{KaiB}$ and $\mathrm{KaiC}$ protein occurs 4-6 hours after peak mRNA abundance, whereas KaiA levels remain relatively constant throughout the circadian cycle (Xu et al. 2000). The kai genes and their protein products exhibit regulatory feedback (Fig. 1) (Ishiura et al. 1998), but this transcriptional/translational regulation appears to be a dispensable layer of reinforcement on a posttranslational clock in the cyanobacterium. Specific cis elements in the kaiA and kaiBC promoters are not required to preserve circadian regulation of gene expression (Xu et al. 2003; Ditty et al. 2005); instead, the cyanobacterial timing mechanism is controlled by posttranslational modifications to the KaiC protein.

An oscillation in KaiC phosphorylation occurs in the presence of only KaiA, KaiB, KaiC, and ATP in a temperature-compensated circadian rhythm in vitro (Nakajima et al. 2005). The beautiful simplicity of this test tube oscillator demonstrates the importance of the Kai-based posttranslational timing circuit and, along with promoter-replacement experiments in vivo, dismisses the requirement for the transcriptional/translational regulatory feedback loop in the cyanobacterial system. 


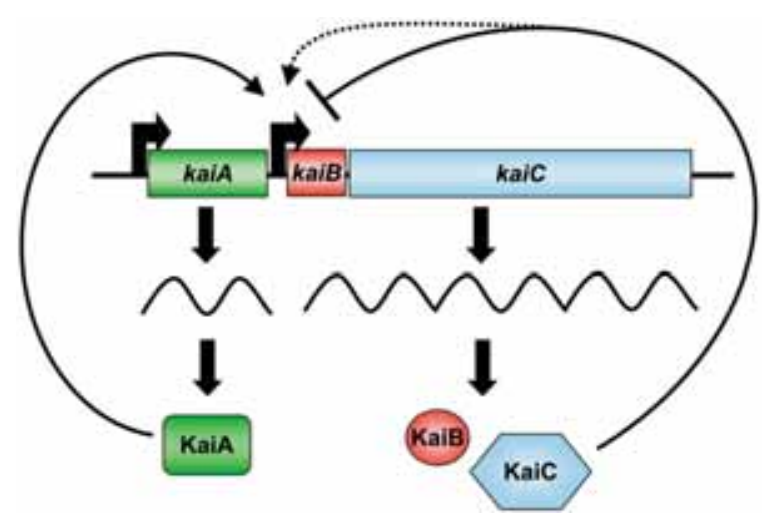

Figure 1. Regulation of the kai locus. Transcription of the kaiA and $k a i B C$ genes is under the control of the circadian clock with peak expression levels that occur at subjective dusk under constant light conditions. One promoter (bent arrow) drives expression of the kaiA gene, and another drives transcription of both the $\mathrm{kaiB}$ and $\mathrm{kaiC}$ genes to create a kaiBC dicistron. Overexpression of KaiA causes an increase in the level of expression of $k a i B C$, whereas overexpression of KaiC inhibits its own expression; together, these data suggest that KaiA and $\mathrm{KaiC}$ are positive and negative regulators, respectively, of the $k a i B C$ promoter. A basal level of $\mathrm{KaiC}$ is required for its own expression (dotted line), which implies an activating role for KaiC in maintaining kaiBC levels. However, this transcriptional regulation and any specific cis elements within the kai promoters are not required for proper clock function and are likely an additional layer of reinforcement to a posttranslational oscillator. (Arrows and perpendicular lines) Positive and negative regulation, respectively.

KaiC forms an ATP-dependent homohexamer and autophosphorylates at adjacent serine and threonine residues (Pattanayek et al. 2004; Xu et al. 2004). The phosphorylation state of KaiC fluctuates in a circadian manner in vivo, yet this progressive phosphorylation of KaiC does not appear to lead to its rapid degradation (Iwasaki et al. 2002). During morning, KaiA dimers enhance the autophosphorylation of KaiC; KaiA and phosphorylated KaiC (KaiC P) form a complex to maintain elevated levels of KaiC $\sim \mathrm{P}$ for the remainder of the day. At night, KaiB joins the KaiA/KaiC $\sim \mathrm{P}$ complex to negate the positive effect of KaiA on KaiC autophosphorylation and accelerate the dephosphorylation of KaiC $\sim \mathrm{P}$ to KaiC. The complex disassociates into its components before dawn and the cycle starts over for the next day (Fig. 2) (for review, see Golden and Canales 2003; Iwasaki and Kondo 2004; Williams 2006).

Input. No true photoreceptor has yet been identified in S. elongatus that is dedicated to transducing external light information to the central oscillator. Instead, the cyanobacterial clock system is sensitive to changes in cellular redox state, which reflects the flux of light driving photosynthesis (Fig. 3). The light-dependent period (LdpA) protein contains iron-sulfur clusters that allow LdpA to detect changes in the redox state of the cell, which are interpreted as changes in light intensity, to modulate the period of the internal oscillation (i.e., obey Aschoff's rule) (Katayama et al. 2003; Ivleva et al. 2005). Cells that lack $l d p A$ maintain the shorter period associated with high light, regardless of actual light intensity.

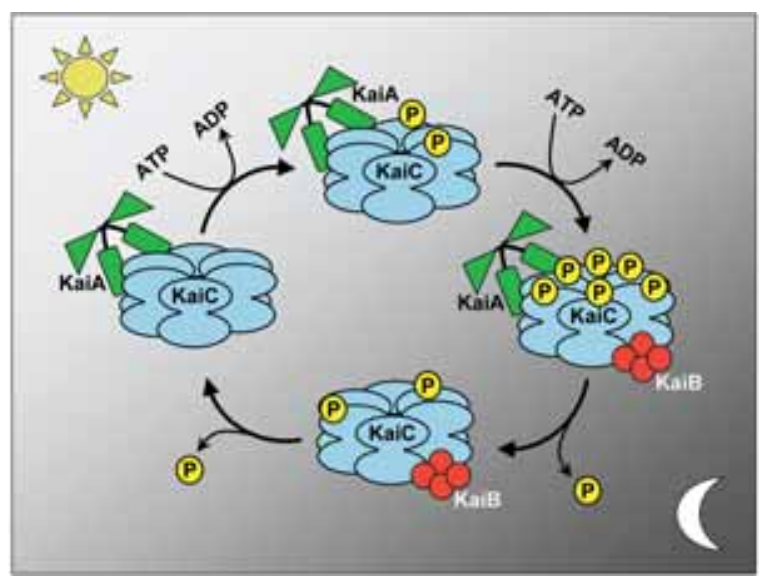

Figure 2. A posttranslational Kai oscillator. Daily rhythms in KaiC phosphorylation are aided by interactions with KaiA and KaiB. During the day, KaiA associates with KaiC to stimulate the autophosphorylation of KaiC. Near subjective dusk, KaiC is in a hyperphosphorylated state and is thought to undergo a conformational change that allows it to have a higher binding affinity for KaiB. Interaction with KaiB during the night results in the dephosphorylation of KaiC. Once in its hypophosphorylated state at subjective dawn, KaiC will again interact with KaiA and the cycle begins anew. (Reprinted, with permission, from Mackey and Golden 2007 [@ Elsevier].)

Resetting the phase of the rhythm in response to abrupt external stimuli, such as pulses of darkness or temperature, occur through the circadian input kinase (CikA) protein (Schmitz et al. 2000). Accumulation of CikA occurs during the night (Ivleva et al. 2006), and low levels of CikA protein are present at higher light intensities. Levels of CikA protein are locked at the low level that is associated with high light in strains deficient in $\operatorname{ldpA}$ (Ivleva et al. 2005). Degradation of CikA can be stimulated by the direct binding of a quinone analog 2,5-dibromo-3methyl-6-isopropyl-p-benzoquinone (DBMIB) to the pseudo-receiver domain of the protein (Ivleva et al. 2006). DBMIB affects electron transport in the photosynthetic apparatus to produce an excess of electrons (reduction), which reflects the redox state of the cell. These results further implicate cellular metabolism and redox state as important factors for clock synchronization.

The period extender (Pex) protein binds to the kaiA promoter and likely represses expression of kaiA (Fig. 3) (Kutsuna et al. 1998; Takai et al. 2006a). Pex delays the internal oscillation when cells are subjected to a light/dark cycle in order to remain in-phase with that cycle (Takai et al. 2006a). Pex accumulates during the night and is undetectable in the light, which suggests that the repression of kaiA expression also has a role in delaying the phase of the oscillation in natural daily cycles.

Both CikA and LdpA have been shown to be part of the Kai protein complex (along with the output protein SasA, discussed below), and at least CikA influences the phosphorylation state of KaiC (Ivleva et al. 2005, 2006). The assembly and disassembly of this large heteromultimeric protein complex, termed the "periodosome," appear to be a driving force behind the cyanobacterial circadian system as a whole. 


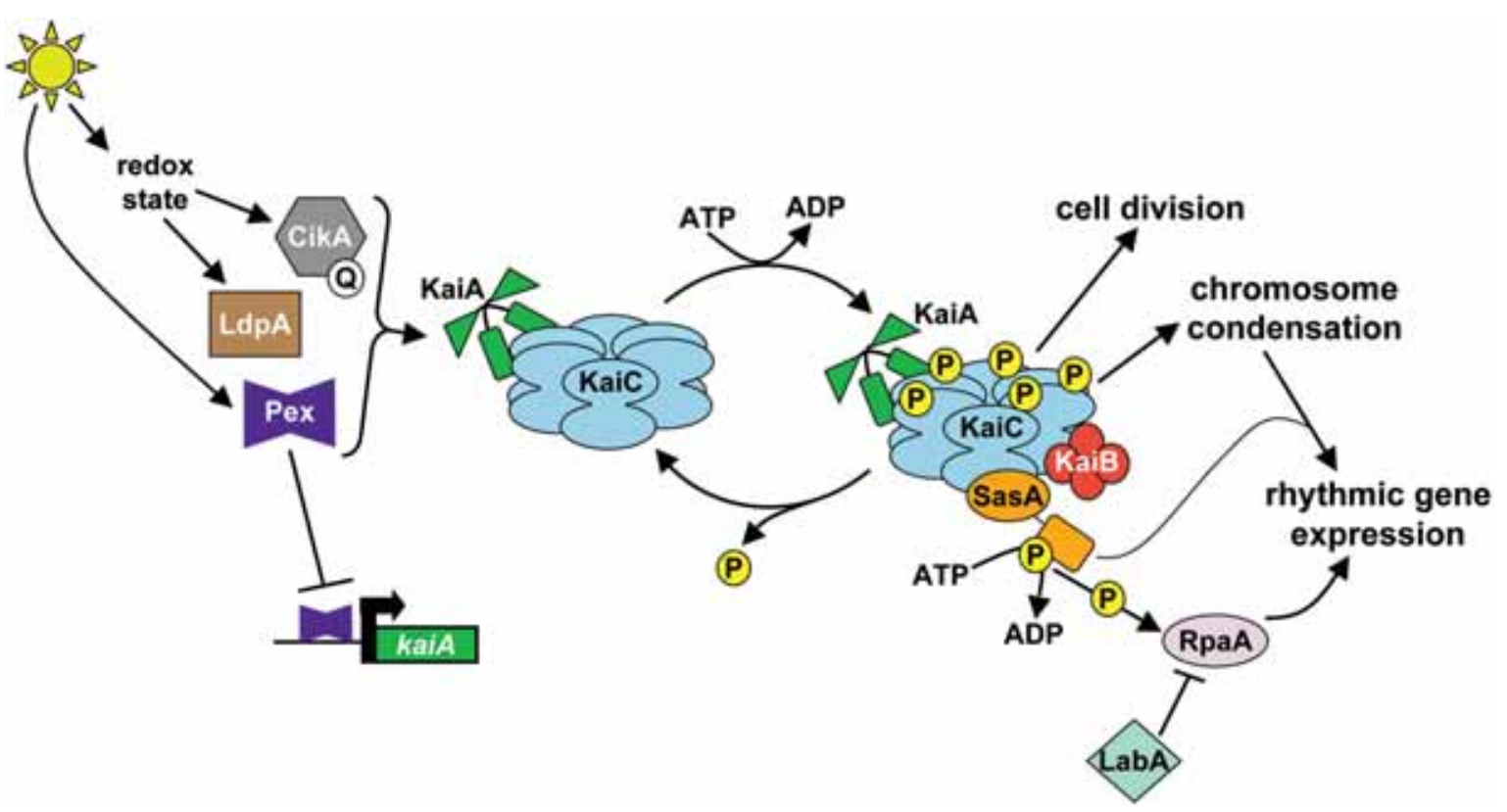

Figure 3. A clock model for the S. elongatus system. The Kai-based posttranslational oscillator is synchronized with the external light/dark cycle through input pathway components CikA, LdpA, and Pex. Changes in cellular redox state, which changes with light quantity, is sensed via iron-sulfur clusters of LdpA and direct binding of a quinone $(\mathrm{Q})$ to CikA. Pex is predicted to repress the expression of kaiA through direct binding of the kaiA promoter. The oscillation of KaiC phosphorylation is achieved through the conflicting actions of KaiA, which stimulates KaiC autokinase activity, and KaiB, which decreases the positive effect of KaiA. Temporal information is transduced from KaiC to SasA. SasA transfers its phosphoryl group to RpaA, which is predicted to activate RpaA; LabA is thought to repress the activity of RpaA. Together, the S. elongatus clock controls many cellular activities, including cell division, chromosome condensation, and rhythmic gene expression. (Arrows and perpendicular lines) Positive and negative regulation, respectively. (Reprinted, with permission, from Mackey and Golden 2007 [@ Elsevier].)

Output. The cyanobacterial circadian clock regulates global gene expression, such that every promoter tested drives expression of luciferase reporter genes in a 24-hour rhythm (for review, see Johnson 2004; Woelfle and Johnson 2006). This global control is likely the result of the Kai-dependent circadian rhythm in chromosome compaction that alters the availability of promoter regions to transcriptional machinery (Fig. 4) (Smith and Williams 2006). Synechococcus adaptive sensor (SasA) receives temporal information through direct interaction with KaiC in the periodosome complex, which stimulates the autophosphorylation of SasA (Fig. 3) (Smith and Williams 2006). Active, phosphorylated SasA transfers its phospho- ryl group to the regulator of phycobiliosome-associated ( $\mathrm{RpaA}$ ) protein, which is predicted to act as a transcription factor to affect expression of downstream clock-controlled genes (Takai et al. 2006b). SasA is not required for rhythmic chromosome compaction, but it is necessary for information generated by the compaction to be translated into rhythmic promoter activity (Smith and Williams 2006).

The SasA/RpaA signal transduction pathway acts as the activation pathway; removal of either component lowers the overall levels of KaiC and decreases expression of bioluminescence reporters (Takai et al. 2006b). The lowamplitude and bright (LabA) protein is proposed to act in a repression pathway to feed temporal information

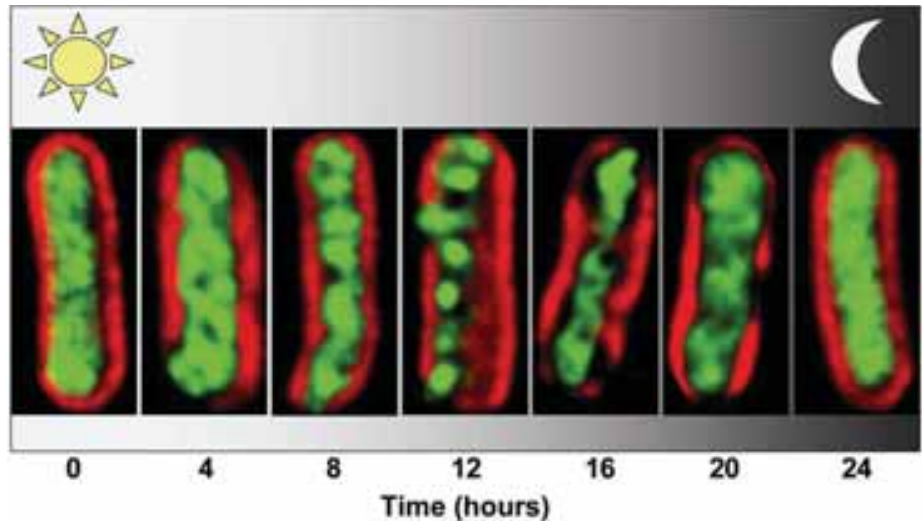

Figure 4. Chromosome compaction in S. elongatus. Deconvolved fluorescence microscopy images. (Red) Autofluorescence from S. elongatus; (green) DAPI-stained DNA of $S$. elongatus cells. Samples were taken at the time indicated on the $Y$ axis during a 12 hours: 12 hours light/dark cycle. The DNA begins in as decompacted chromosomes at 0 hour, arranges into distinct "nucleoid" regions in anticipation of the light-to-dark transition (12 hours), and returns to a diffuse state at 24 hours. (Figure courtesy of R.M. Smith and S.B. Williams, Department of Biology, University of Utah, Salt Lake City.) 
through RpaA in an output pathway separate from that of SasA (Taniguchi et al. 2007). The connection between LabA and RpaA is likely indirect.

The cyanobacterial circadian clock persists in cells with doubling times much shorter (as fast as 6 hours) than a full circadian cycle (Kondo et al. 1997); yet, the population of cells remains in-phase with one another through unknown mechanisms (Mihalcescu et al. 2004). Cell division is gated by the endogenous circadian rhythm, such that there is a "forbidden" phase that stretches from late day to early-to-midnight (Mori et al. 1996), which is coincident with the formation of the periodosome and with the transition from a decondensed to condensed nucleoid.

S. elongatus does not fix nitrogen (Herrero et al. 2001), and without the need to separate photosynthesis and nitrogen fixation, the selective pressure to possess a robust circadian oscillator that regulates transcription is less obvious. Nonetheless, in a competitive environment, $S$. elongatus cells with a free-running period (FRP) that closely matches that of the external light/dark cycle possesses a growth advantage over strains whose FRP differs from that of the external cycle and over strains that lack a functional clock; however, the growth rates of cultures maintained individually are indistinguishable, even in different light/dark cycles (Ouyang et al. 1998).

\section{EUKARYOTIC CIRCADIAN CLOCK SYSTEMS}

Although the end result of maintaining an internal timing mechanism is the same for both prokaryotic and eukaryotic organisms, many differences exist between the two broad categories. Regulation of transcription by the clock is widespread, yet the global regulation of transcription from all promoters is unique to the cyanobacterium. Likewise, posttranslational mechanisms are key regulators in maintaining circadian timing in each system, yet the posttranslational oscillator of S. elongatus is unique and will likely remain unique given the importance of promoter elements in clock gene function in the eukaryotic systems.

One main difference is the means by which oscillators are reset in response to changes in the daily environment. Photoreceptors dedicated to receiving light information for the oscillator have not been identified in cyanobacteria; instead, the cells appear to interpret changes in cellular redox state as an indirect measurement of light intensity. The eukaryotic organisms for which the molecular mechanisms of the endogenous oscillator have been described possess photoreceptors that sense light to directly influence the steady-state level of core oscillator components to alter the phase angle of the rhythm.

The presence of a nucleus in eukaryotic organisms provides, perhaps not surprisingly, another critical difference in their internal timing mechanisms as compared to the prokaryotic system. Compartmentalization and translocation between the cytoplasm and nucleus are critical processes in the progression of the eukaryotic clock. The internal oscillators of eukaryotic systems are controlled by a common mechanism: transcriptional/posttranslational regulatory feedback loops (Fig. 5) (for review, see BellPedersen et al. 2005). In these loops, genes that encode the positive effectors are transcribed and their protein products translocate to the nucleus to activate transcription of genes that encode the negative elements. As the concentration of the negative elements increases, they inhibit the activity of the positive effectors, which, in turn, decreases the level of expression of the negative elements. Progressive phosphorylation of the negative elements induces degradation, which leads to a decrease in their relative amounts. This decline relieves the inhibition of the positive effector proteins and the cycle can begin anew. An additional feedback loop exists in some oscillators that includes activating and inhibiting elements that compete for availability to the promoter region of (at least) one of the genes that encodes a positive effector protein. The combined, interlocking transcriptional/posttranslational feedback loops within the circadian oscillator require approximately 24 hours to complete one cycle.

\section{Drosophila melanogaster}

A working model of the molecular components of the Drosophila system is depicted in Figure 6 (for review, see Bell-Pedersen et al. 2005; Hardin 2006; Taghert and Shafer 2006).

Oscillator. Two interlocking feedback loops have been described in the Drosophila circadian oscillator. The first loop consists of the positive elements CLOCK (CLK) and CYCLE (CYC), which are transcription factors that contain basic-helix-loop-helix (bHLH) DNA-binding domains, PER-ARNT-SIMS (PAS) dimerization domains, and the negative elements PERIOD (PER) and TIMELESS (TIM). The $C l k$ gene is rhythmically transcribed with peak expression occurring near dawn, whereas cyc mRNA levels remain constant. At midday, CLK/CYC heterodimers bind to E boxes in the promoter regions of the per and tim genes (as well as those of vrille [vri] and PAR domain $1 \varepsilon$ $[P d p l \varepsilon]$, discussed below) to activate their transcription. The four protein products of these genes influence the activity of CLK protein and Clk expression levels to create the regulatory feedback loops necessary for generating 24hour internal time.

Activation of per and tim expression by CLK/CYC leads to increased mRNA levels with peak accumulation occurring during the early evening. However, peak protein levels do not occur until late night due to the phosphorylation-induced degradation of PER protein by the kinase DOUBLE-TIME (DBT). DBT is a homolog of casein kinase $1 \varepsilon(\mathrm{CK} 1 \varepsilon)$ in mammals (discussed below). Dephosphorylation of PER occurs by protein phosphatase 2A (PP2A). These opposing activities alter PER stability, which is also dependent on its interactions with DBT and TIM. The PER/DBT complex binds TIM, which stabilizes PER by blocking DBT-dependent phosphorylation. Nuclear translocation of PER/DBT and TIM occurs independently and is promoted by casein kinase 2 (CK2) and Shaggy (SGG), which phosphorylate PER and TIM, respectively. After nuclear translocation, PER/DBT and PER/DBT/TIM complexes can inhibit CLK function through direct interaction and DBT-dependent phosphorylation of CLK, which helps to release the CLK/CYC het- 


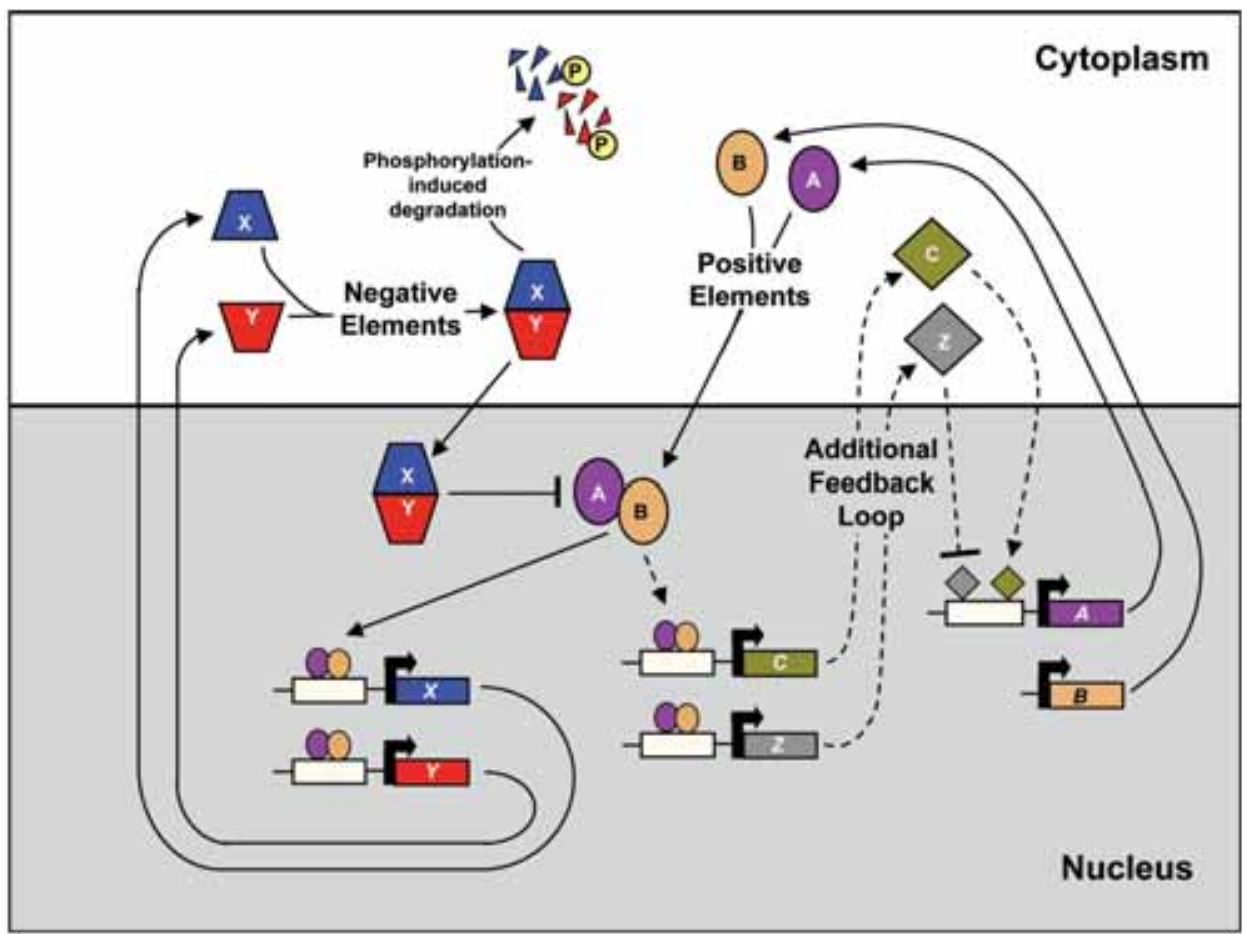

Figure 5. Transcription/translation regulatory feedback loops: A common theme in the generation of eukaryotic circadian rhythms. Many circadian systems are composed of positive and negative elements that are involved in feedback loops. Positive elements (ovals) interact and bind to upstream elements (white rectangles) in the promoter regions (bent arrows) of genes that encode negative elements to activate their expression. These negative components (tetrahedrons) interact to inhibit the activity of the positive elements, which ultimately leads to a decrease in expression of the negative elements. Progressive phosphorylation $(P)$ of negative elements leads to their degradation and a relief of the inhibition of the positive effectors to allow the cycle to start again. Other components (diamonds) may be present that bind to the promoters of genes that encode the positive elements to comprise an additional feedback loop. (Arrows and perpendicular lines) Positive and negative regulation, respectively.

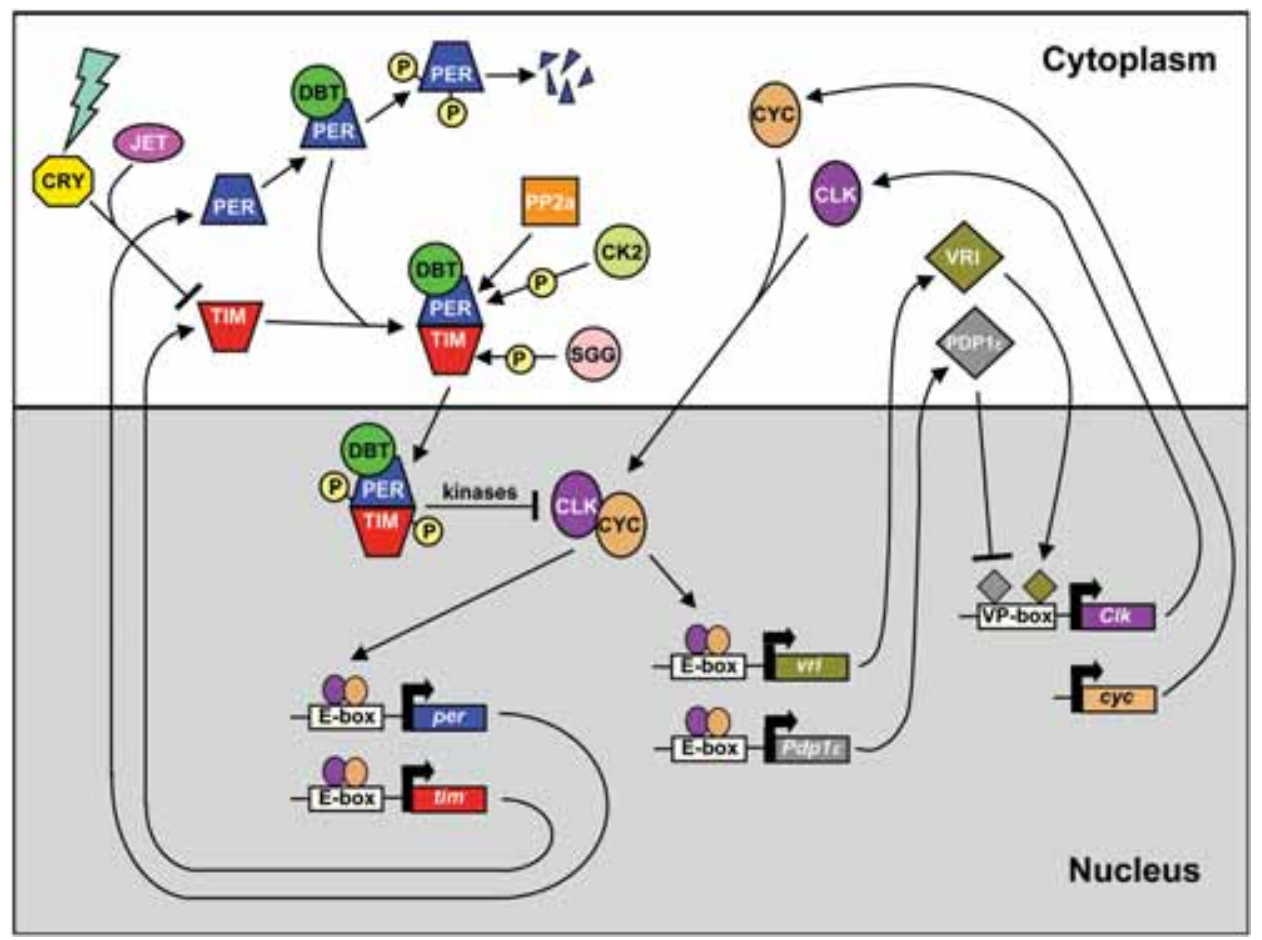

Figure 6. Model for the circadian clock of D. melanogaster. See Figure 5 for basic mechanism and text for details specific to the Drosophila oscillator. (Vertical ovals) Positive elements CLK and CYC; (white rectangles) E-box elements; (bent arrows) promoters; (tetrahedrons) negative elements PER and TIM; (circles) kinase proteins DBT, SGG, and CK2; (square) PP2a phosphatase; (octagon) CRY; (horizontal oval) JET; (diamonds) additional feedback components VRI and PDP1E. (P) Phosphorylation. (Arrows and perpendicular lines) Positive and negative regulation, respectively. 
erodimers from the E-box promoter sequences and reduce expression of per and tim (as well as that of $v r i$ and $P d p 1 \varepsilon$ ). The progressive phosphorylation of PER by DBT ultimately results in the degradation of PER and the trough in its protein accumulation occurs in early morning. In contrast, there is a rhythm in the phosphorylation of CLK protein, but the overall protein level does not cycle. Coincident with the timing of PER degradation, TIM protein levels decrease due to light-induced degradation via the blue light photoreceptor CRYPTOCHROME (CRY). With the PER/DBT/TIM inhibition of CLK lifted and new CLK protein being made, the cycle begins anew.

Additional regulation of the Clk locus is thought to occur through a second feedback loop via the VRI and PDP1ع bZIP transcription factor proteins, as well as a predicted constitutive activator protein. In the nucleus, CLK/CYC heterodimers activate transcription of vri and $P d p 1 \varepsilon$ with peak mRNA accumulation occurring in the early night and midnight, respectively. VRI protein accumulates quickly in the cytoplasm, enters the nucleus to bind the VRI/PDP1ع box (VP box) in the Clk promoter, and inhibits $C l k$ transcription. As levels of VRI protein dis-

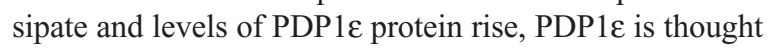
to displace VRI from the VP box to enhance expression of $C l k$ in the late night (which ultimately results in peak $C l k$ mRNA levels at dawn).

Input. Entrainment of the Drosophila clock is mediated through (at least) the CRY-dependent degradation of TIM (Ceriani et al. 1999; Emery et al. 2000). In constant darkness, when TIM protein levels are rising, brief exposure to light results in a decrease in TIM protein to trough levels and an overall phase delay, whereas a light pulse during the time when TIM protein is declining causes a phase advance. Light exposure causes a conformational change in CRY that allows it to bind TIM. TIM is phosphorylated on at least one tyrosine residue and interacts with the JETLAG (JET) protein, which targets TIM for ubiquitination and degradation by the proteasome pathway (Koh et al. 2006).

Output. The regulatory feedback loops that comprise the molecular oscillator in flies is found in various tissues (e.g., head, wings, legs, and antennae), each of which appear to function autonomously and can be directly reset by light (for review, see Bell-Pedersen et al. 2005). These oscillators control pupal eclosion, courtship behavior, locomotor activity behavior, and olfactory responses. Although there does not appear to be a hierarchy of "master" and "slave" pacemakers, there may be communication among oscillators in the brain through the neuropeptide pigment-dispersing factor (PDF), which is secreted in the dorsal brain with a circadian pattern (for review, see Taghert and Shafer 2006).

\section{Mammals}

The current model of the molecular components of the mammalian clock system is portrayed in Figure 7 (for review, see Gachon et al. 2004; Ko and Takahashi 2006; Kuhlman and McMahon 2006).
Molecular oscillator. Like the Drosophila system, the mammalian clock is described as having two interlocking regulatory feedback loops. In the first loop, the positive effectors are CLOCK and BMAL1 (brain and muscle ARNT-like protein 1), which is the mammalian homolog of Drosophila's CYC. CLOCK and BMAL1 are bHLHPAS domain-containing transcription factors that form heterodimers in the cytoplasm, enter the nucleus, and bind to the E-box sequence in the promoters of the genes that encode the negative elements. BMAL1 contains both nuclear localization signals and nuclear export signals that allow it to shuttle between the nucleus and cytoplasm to promote the nuclear translocation of CLOCK (Kwon et al. 2006). In mammals, the negative limb of this feedback loop consists of some combination of the Period (in mice, Perl, Per2, and Per3) and Cryptochrome (Cryl and Cry2) genes. To date, the role of a mammalian protein that is similar in sequence to Drosophila's TIM has not been firmly established in the generation of circadian rhythms. Instead, the CRY proteins in mammals have taken on the role of Drosophila's TIM; CRYs form heterodimers with the PER proteins, enter the nucleus, and inhibit the activity of CLOCK/BMAL1 complexes. Without CLOCK/BMAL1 to activate transcription of the Per and Cry genes, levels of $P e r$ and $C r y$ transcripts, and their respective protein products, decline. Degradation of PER and CRY is induced by progressive phosphorylation by casein kinase $1 \delta(\mathrm{CK} 1 \delta)$ and $\mathrm{CK} 1 \varepsilon$, which leads to a release of the inhibition of CLOCK/BMAL1, and the cycle starts over. Interestingly, in contrast to Drosophila, the photoreceptor properties of CRY proteins in mammals have not yet been demonstrated.

A second feedback loop regulates the expression of the Bmall gene. CLOCK/BMAL1 heterodimers enter the nucleus and bind to the E-box sequences in the promoter regions of genes that encode the retinoic-acid-related orphan nuclear receptors REV-ERB $\alpha$ and $\mathrm{ROR} \alpha$, which compete for the ROR element (RORE) in the Bmall promoter. The ROR family ( $\alpha, \beta$, and $\gamma$ ) of proteins activates Bmall expression, and REV-ERB $\alpha$ and REV-ERB $\beta$ repress expression of Bmall. This regulation of Bmall maintains a robust rhythm of activity in vivo. Proteins with sequences similar to those of the ROR and REVERB families exist in Drosophila, but whether they participate in the clock system is not yet known.

SCN as master pacemaker. In contrast to the autonomous oscillators that exist in Drosophila cells and tissues, the oscillations that exist in tissues in the mammalian circadian clock system are arranged in a hierarchy. The suprachiasmatic nucleus (SCN) in the anterior hypothalamus of the brain is the "master" pacemaker. Removal of the SCN results in a loss of rhythmic outputs, including sleep/wake cycle, wheel-running behavior, hormone production, core body temperature, and accumulation of clock-controlled mRNA and protein. The master pacemaker in the SCN is reset daily by light signals that are transmitted through the retinohypothalamic tract and retinal ganglion cells to cause cyclic-AMP response element binding (CREB) protein-mediated induction of Perl transcription (discussed in Part IB). The SCN then transmits these environmental timing cues to the peripheral "slave" 


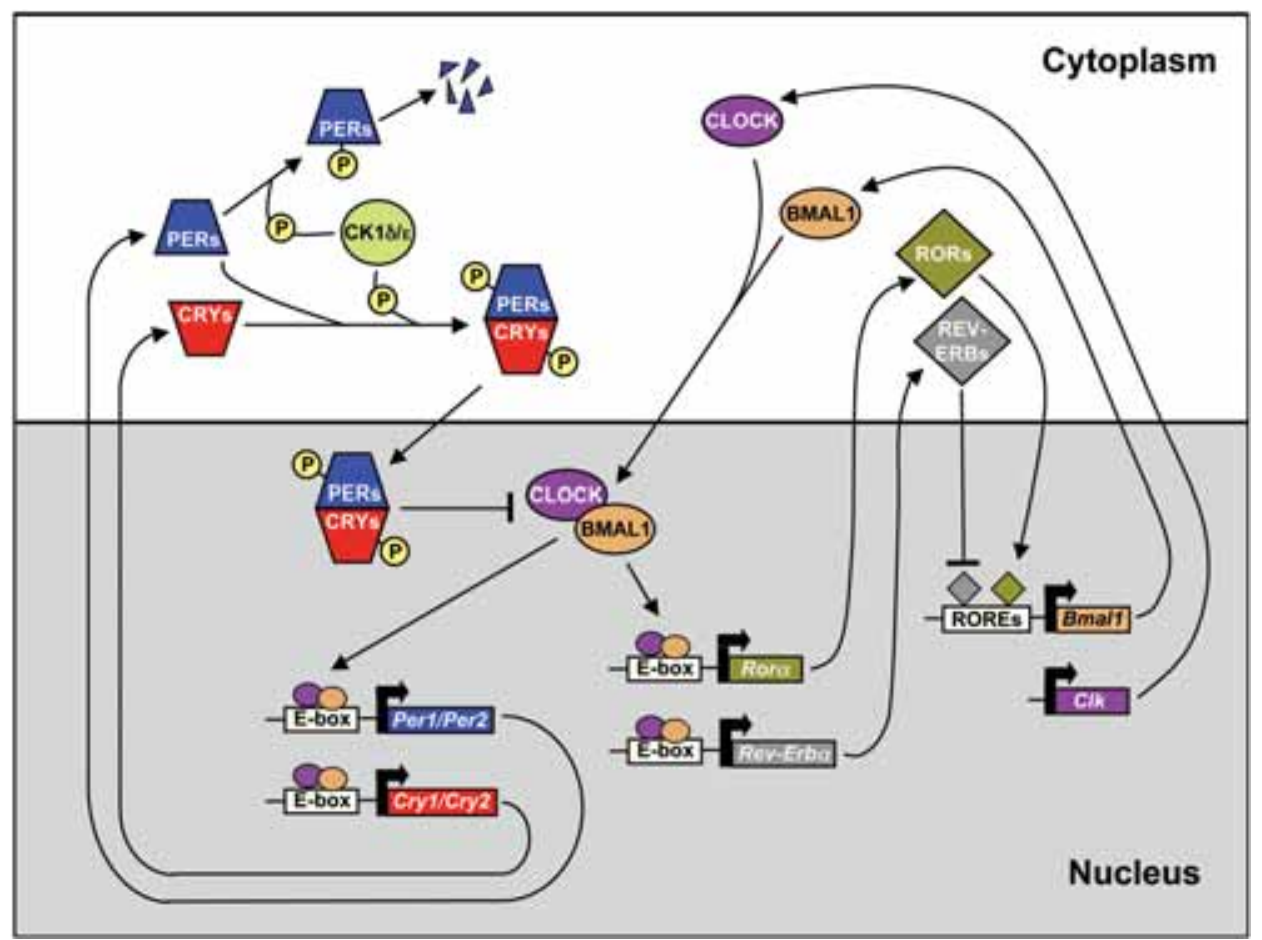

Figure 7. Model for the circadian clock in mammals. See Figure 5 for basic mechanism and text for details specific to the mammalian oscillator. (Horizontal ovals) Positive elements CLOCK and BMAL1; (white rectangles) E-box elements; (bent arrows) promoters; (tetrahedrons) negative elements PERs and CRYs; (circle) kinase proteins CK1 $\delta$ and CK1 $\delta$; (diamonds) additional feedback components RORs and REV-ERBs. $(P)$ Phosphorylation. (Arrows and perpendicular lines) Positive and negative regulation, respectively.

oscillators in mammalian tissues via polysynaptic and humoral pathways to coordinate and synchronize their oscillations with that of the master SCN oscillator (discussed in Part IB).

Output via histone modification. Transcriptional activation of targets of the CLOCK/BMAL1 heterodimer

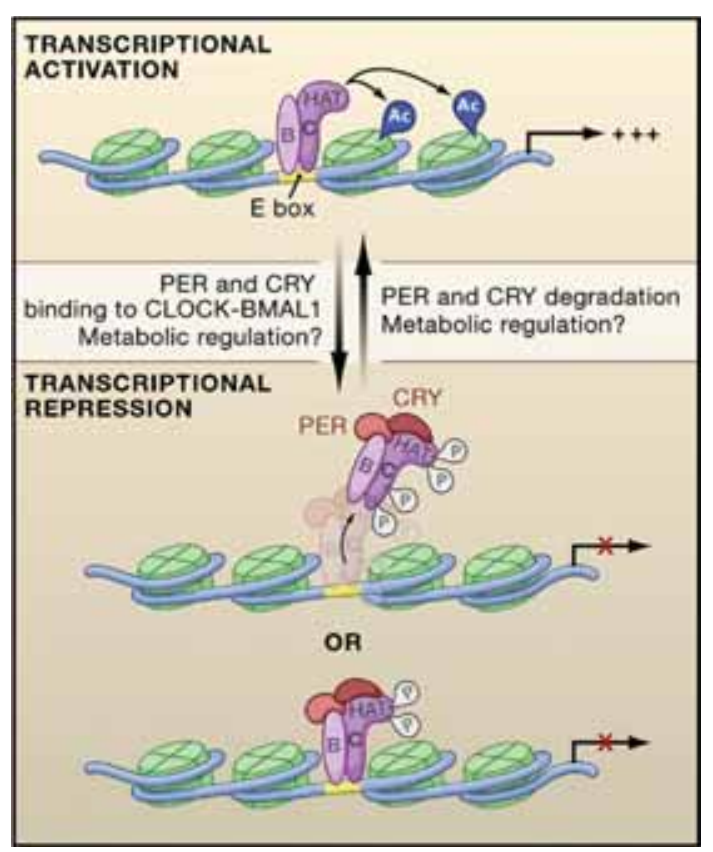

results from the histone acetyltransferase (HAT) activity of CLOCK itself (Doi et al. 2006). Histone acetylation promotes transcription through the modification of histones to allow for the "opening" of condensed chromatin, which provides access to the transcriptional machinery (Fig. 8). The HAT activity of CLOCK is necessary for the activation of transcription of clock genes Per and Cry and is thus

Figure 8. Model for the regulation of rhythms in CLOCKBMAL1-dependent transcription. Transcriptional activation requires E-box binding by CLOCK(C)-BMAL1(B) heterodimers and CLOCK histone acetyltransferase (HAT)-dependent acetylation of histone $\mathrm{H} 3$ (Ac). Transcriptional repression is mediated by several events. Frist, PER and CRY bind to CLOCK-BMAL1. Formation of this complex can inhibit CLOCK HAT activity by promoting CLOCK phosphorylation (P) or inducing a conformation change in CLOCK-BMAL1. Binding of PER and CRY to CLOCK-BMAL1 may or may not cause the dissociation of the complex from $\mathrm{E}$ boxes. In either case, loss of CLOCK HAT events start with the degradation of PER and CRY. Phosphorylated CLOCK is then either dephosphorylated or degraded and resynthesized. CLOCK forms heterodimers with newly synthesized BMAL1, this complex binds to E boxes, and CLOCK HAT acetylates histones to activate transcription. The metabolic state of the cell may also modulate transcriptional activity by promoting either transcriptional activation or repression. (Figure and legend reprinted, with permission, from Hardin and Yu 2006 [@ Elsevier].) 
believed to be essential for the generation and maintenance of endogenous circadian timing in mammals. The sequence similarity among CLOCK homologs in other mammals and insects (including Drosophila) hints at a conserved mechanism for transcriptional regulation of core clock genes by posttranslational histone modifications.

\section{Neurospora crassa}

The current model for the internal timekeeping mechanism of Neurospora crassa is depicted in Figure 9 (for review, see Dunlap and Loros 2006; Liu and BellPedersen 2006; Vitalini et al. 2006).

Oscillator. The oscillator of the Neurospora system consists of a single, well-characterized feedback loop composed of the positive elements WHITE COLLAR-1 (WC-1) and WHITE COLLAR-2 (WC-2) and the negative element FREQUENCY (FRQ). The positive elements are PAS-domain-containing, GATA-type transcription factors encoded by the $w c-1$ and $w c-2$ genes. Neither $w c-1$ nor $w c-2$ mRNA abundance accumulates in a rhythmic fashion, yet WC-1, but not WC-2, protein levels fluctuate with a near 24-hour period. WC-1 and WC-2 proteins heterodimerize in the cytoplasm to form the WHITE COLLAR COMPLEX (WCC). This complex translocates to the nucleus and binds directly to the Clock box (C box) of the frq promoter to activate frq expression. The level of frq mRNA increases and peaks midday; peak levels of FRQ protein occur 4-6 hours after peak mRNA levels. FRQ protein forms homodimers that complex with the FRQinteracting RNA helicase (FRH). Stability of FRQ is determined by the conflicting actions of numerous kinases and phosphatases. The kinase proteins casein kinase I and II (CKI, CKII), calcium/calmodulin-dependent protein kinase 1 (CAMK1), and Period-4 (PRD-4) progressively phosphorylate FRQ, which facilitates the interaction of hyperphosphorylated FRQ with the ubiquitin ligase FWD1 to target FRQ for degradation; the protein phosphatases 1 (PP1) and 2A (PP2A) dephosphorylate FRQ to allow for its accumulation. The FRQ/FRH complex enters the nucleus to inhibit the action of the WCC through phosphorylation. The hyperphosphorylated WCC is inactive and no longer capable of supporting transcription from the frq promoter. The decrease in frq mRNA level, along with the targeted degradation of FRQ protein and dephosphorylation of the WCC by PP2A, results in a reactivation of frq transcription to allow the cycle to begin again. It is unclear how these events are coordinated. FRQ protein also has a role (directly or indirectly) in the positive regulation of the WC proteins by stabilizing $w c-2$ mRNA and up-regulating WC-1 protein levels through a posttranscriptional mechanism (not shown in Fig. 9); this positive feedback loop is thought to add stability and robustness to the Neurospora internal timing oscillation. FRQ, however, is not required for the rhythmic accumulation of $\mathrm{WC}-1$ protein. The basis of the WC- 1 cycle is unclear and may be related to a second oscillator.

At least one additional circadian oscillator exists in Neurospora that does not require the FRQ protein, i.e., a

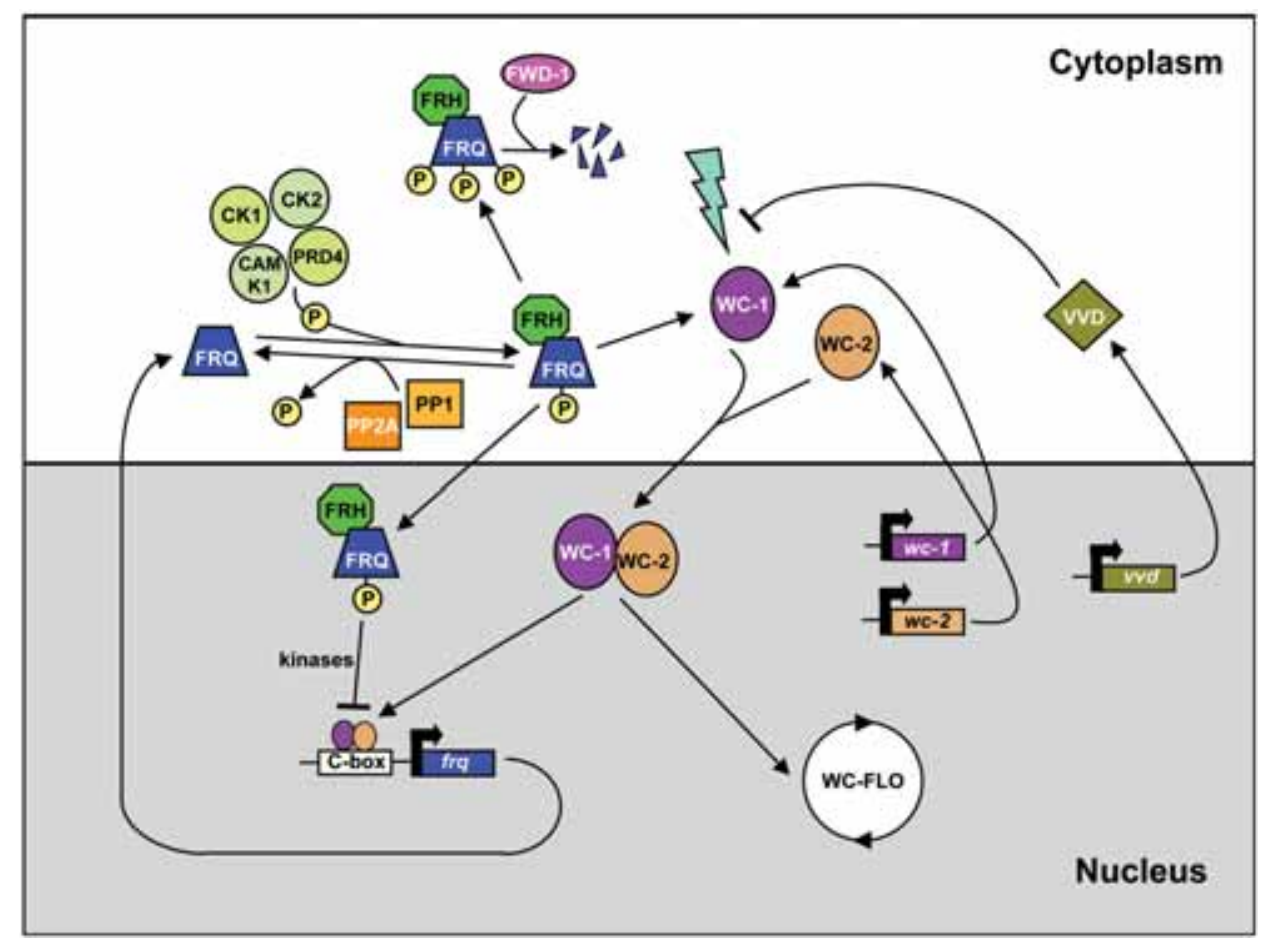

Figure 9. Model for the circadian clock in $N$. crassa. See Figure 5 for basic mechanism and text for details specific to the Neurospora oscillator. (Vertical ovals) Positive elements WC-1 and WC-2; (white rectangles) Clock-box elements; (bent arrows) promoters; (tetrahedron) negative element FRQ; (circles) kinase proteins CK1, CK2, CAMK1, and PRD4; (squares) PP1 and PP2a phosphatases; (octagon) FRH; (horizontal oval) FWD-1; (diamond) additional feedback component VVD. ( $P$ ) Phosphorylation. (Arrows and perpendicular lines) Positive and negative regulation, respectively. 


\section{BIOLOGICAL RHYTHMS WORKSHOP IA}

FRQ-less oscillator (FLO). The WC-1 and WC-2 proteins function in one FLO to form the WC-FLO (Fig. 9). This oscillator controls temperature-compensated molecular rhythms in mRNA accumulation of at least one clockcontrolled gene, $c c g-16$, with a period near 24 hours under constant conditions in the absence of frq.

Input. Time-of-day information can be perceived by the Neurospora circadian system through light or temperature cues. WC-1 bound to the flavin FAD serves as a blue light photoreceptor that interacts with WC-2 to form a "light-response WC complex" that binds to light-response elements in the promoters of light-regulated genes, including those in the frq promoter, to activate their transcription. The abrupt change in the frq transcript is reflected in protein accumulation such that a light pulse during the time when the frq transcript is increasing will cause a phase advance (Fig. 10A), and the same stimulus given when the frq transcript is on the decline will result in a phase delay (Fig. 10B). The protein VIVID (VVD) helps to dampen the light-responsive increase in FRQ protein at dawn in natural light/dark cycles such that the clock can maintain its internal autoregulatory feedback loop during the day and reset its clock at dusk each day by influencing frq mRNA turnover.

Temperature also resets the phase of the Neurospora rhythm through posttranscriptional changes that affect FRQ protein level and protein form (long or short). Absolute levels of FRQ protein are higher at warmer temperatures than at cooler temperatures. In response to a temperature step-up, the current level of FRQ becomes the trough of the "new" rhythm; a temperature step-down results in the current level of FRQ becoming the "new" peak (Fig. 10C). Either condition resets the clock to a new phase as determined by the external temperature.

Output. The overt rhythm of asexual spore formation (conidiation) is the best-characterized output from the Neurospora circadian system. However, many other processes are under clock control including, but not limited to, lipid metabolism, development, $\mathrm{CO}_{2}$ evolution, enzyme activity, and the rhythmic regulation of mRNA and protein accumulation. To date, more than 180 candidate clockcontrolled genes have been identified (Vitalini et al. 2006). Recent work has implicated the clock in regulation of components of cellular mitogen-activated protein kinase (MAPk) signaling pathways that sense and respond to environmental stress. MAPk pathways are ubiquitous in eukaryotic systems and clock control over these universal regulatory systems would allow for numerous downstream genes to be affected in a circadian manner.

\section{Arabidopsis thaliana}

The interlocking molecular feedback loops that comprise the Arabidopsis circadian system are portrayed in Figure 11 (for review, see Mizuno and Nakamichi 2005; Gardner et al. 2006; McClung 2006).

Oscillator. The Arabidopsis circadian oscillator contains (at least) three interconnected feedback loops, each
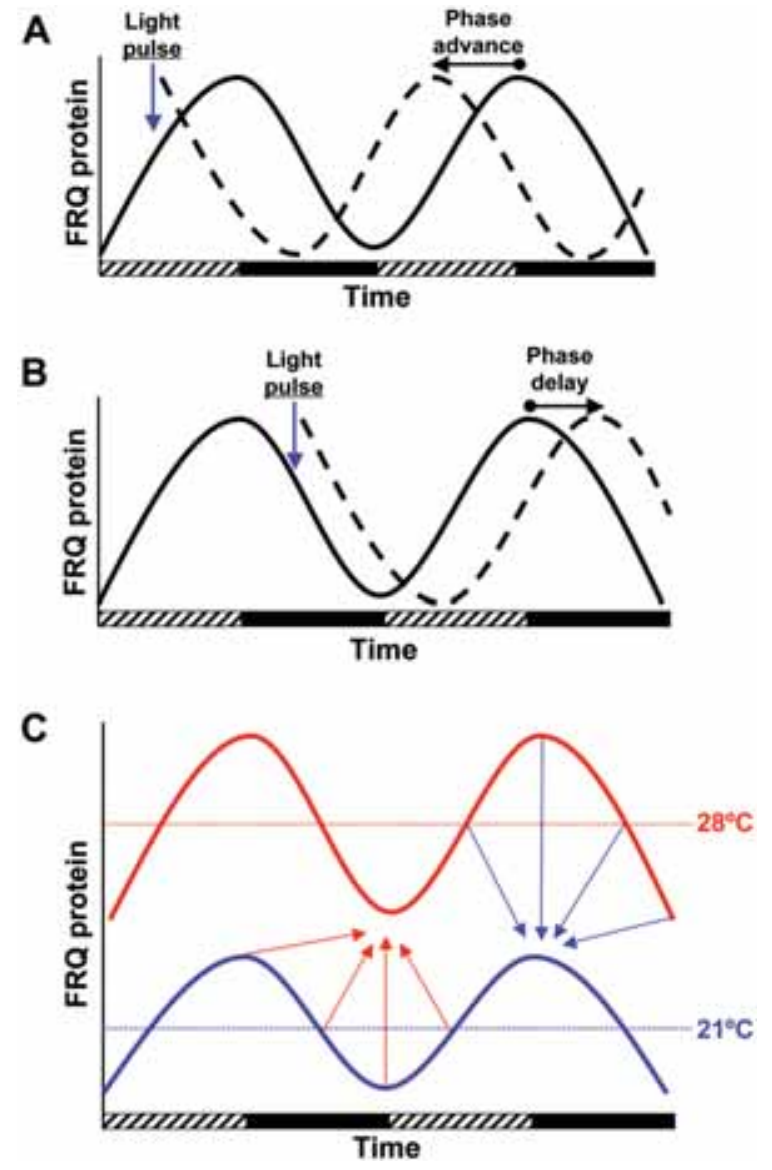

Figure 10. Light and temperature resetting of the $N$. crassa circadian oscillator. FRQ protein levels fluctuate in constant darkness with peak expression levels occurring near the transition between subjective day (hatched bars on $X$ axis) and subjective night (black bars on $X$ axis). Exposure to light causes an increase in the expression of the frq gene and subsequent FRQ protein, such that a light pulse during the time when FRQ protein is rising will cause a phase advance as in $A$ and a light pulse during the time when FRQ protein is declining will cause a phase delay as shown in $B$. (C) FRQ protein levels are higher at warmer temperatures (red curve) than at cooler temperatures (blue curve). After a temperature step-up (from $21^{\circ} \mathrm{C}$ to $28^{\circ} \mathrm{C}$ ), the current level of FRQ becomes the trough of the new rhythm (red arrows). After a temperature step-down (from $28^{\circ} \mathrm{C}$ to $21^{\circ} \mathrm{C}$ ), the current level of FRQ protein becomes the trough of the resulting rhythm (blue arrows).

of which includes the MYB-like transcription factors LATE ELONGATED HYPOTCOTL (LHY) and CIRCADIAN CLOCK ASSOCIATED 1 (CCA1). In the first loop, the positive element TIMING OF CAB EXPRESSION 1 (TOC1; also known as PRR1) activates transcription of $L H Y$ and $C C A 1$, whose protein products translocate to the nucleus to bind to the evening element (EE) of the TOC1 promoter and directly inhibit its expression. As the level in TOC1 mRNA, and subsequently TOC1 protein, declines, CCA1 and LHY levels also decrease, which relieves the inhibition on the TOC1 promoter and the cycle can start again with peak TOC1 abundance occurring at subjective dawn. However, genetic evidence suggests that the TOC $1 /$ CCA1/LHY loop is 


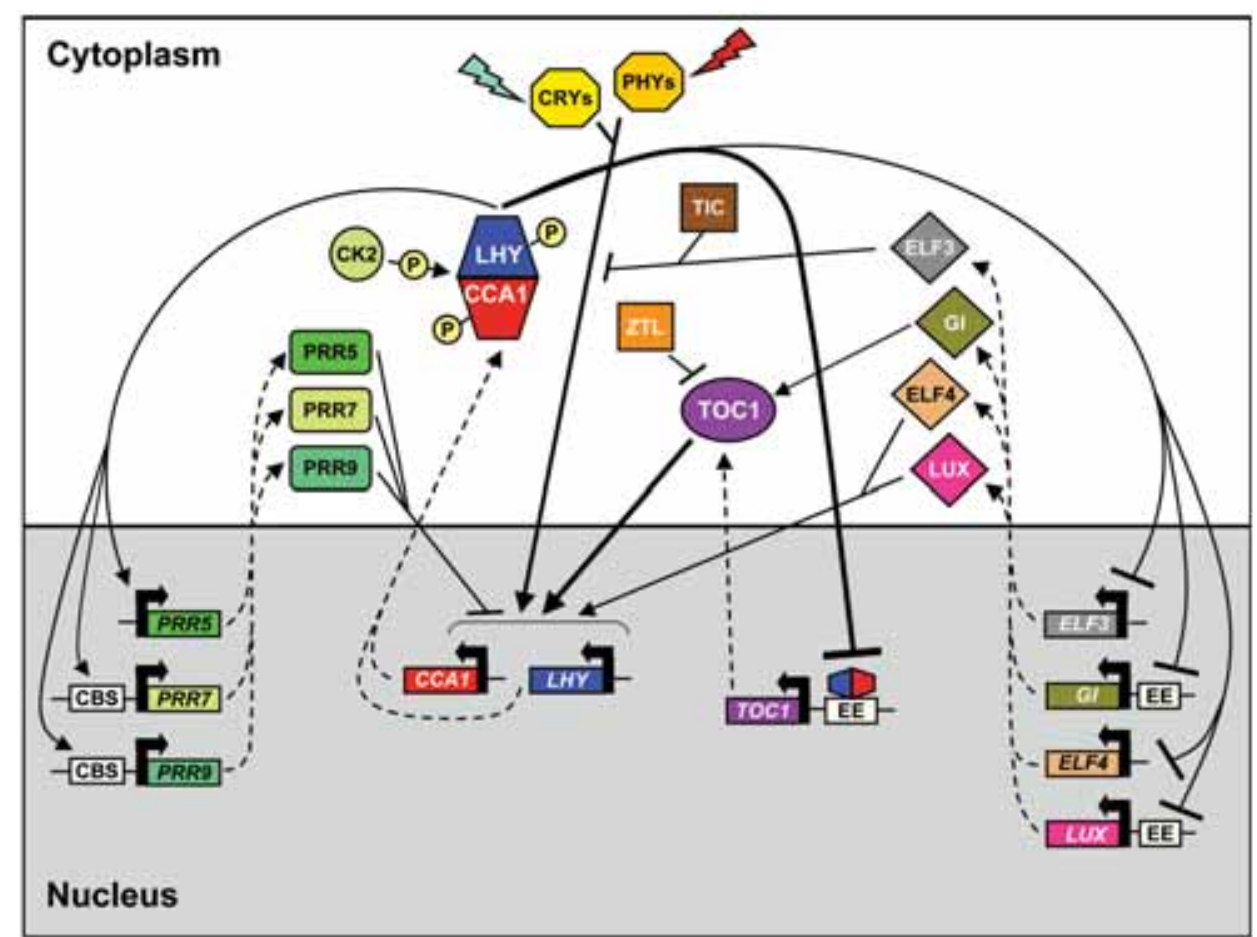

Figure 11. Model for the circadian clock of $A$. thaliana. See Figure 5 for basic mechanism and text for details specific to the three interlocking Arabidopsis feedback loops. Loop 1 is composed of positive element TOC1 (horizontal oval) and negative elements LHY and CCA1 (tetrahedrons) that bind to the evening element (EE; white rectangle) of the TOC1 promoter. Loop 2 includes LHY and CCA1 as positive elements that activate transcription by binding to the CCA1-binding site (CBS; white rectangles) element of the $P R R 5 / 7 / 9$ genes, whose protein products negatively affect $C C A 1$ and $L H Y$ expression. Loop 3 contains LHY/CCA1 negatively inhibiting expression of a subset of genes that encodes the proteins ELF3, GI, ELF4, and LUX (diamonds), which feed back to affect the expression or activity of the LHY, CCA1, and TOC1 proteins. Additional components include CK2 kinase (circle), CRYs and PHYs (octagons), and inhibitory proteins TIC and ZTL (squares). (P) Phosphorylation. (Arrows and perpendicular lines) Positive and negative regulation, respectively.

incomplete and that the GIGANTEA (GI) protein is likely an additional component in this feedback loop that is involved in the activation of TOC1. The promoter of GI contains multiple EEs that would allow for inhibition by LHY/CCA1 to regulate its expression. Both LHY and CCA1 are phosphorylated by casein kinase II (CK2), which is necessary for clock function in vivo, but its role in the degradation of these proteins is not fully understood. LHY is targeted for degradation by the proteins DE-ETIOLATED 1 (DET1) and CONSTITUTIVELY PHOTOMORPHOGENIC 1 (COP1); TOC1 is targeted for degradation by a ZEITLUPE (ZTL)-dependent mechanism.

The second loop includes three pseudo-response regulators (PRR5, PRR7, and PRR9), whose respective proteins have distinct and nonoverlapping roles, but together are involved in the inhibition of $L H Y$ and $C C A 1$, and activation of $T O C 1$ expression. CCA1, in turn, binds directly to the CCA1-binding site (CBS) in the promoters of $P R R 7$ and $P R R 9$ to activate their transcription.

The precise role of the PRR proteins in the clock is still unclear because they appear to be involved in light and/or temperature entrainment in addition to their role in maintaining internal timing. TOC1 (PRR1) likely has a critical role in regulating gene expression because it has been shown to interact with a number of bHLH transcription factors, including PHYTOCHROME INTERACTING FACTOR proteins (PIF3, PIF4) that are known to interact with PHYB and PIF3-LIKE proteins (PIL1, PIL2, PIL5, PIL6) that are involved in light signal transduction.

In a third loop, the MYB transcription factor LUX ARRYTHMO (LUX) activates expression of CCAI and $L H Y$, which bind to the EE in the $L U X$ promoter to inhibit its expression. Additionally, EARLY FLOWERING 4 (ELF4) is required for rhythmic expression of CCA1 and $L H Y$, but not $T O C 1$, and transcription of ELF4 is repressed by CCA1/LHY.

Input. Entrainment of the Arabidopsis clock occurs primarily through light. Red light responses occur through PHYTOCHROME proteins PHYA, PHYB, PHYD, PHYE, and likely PHYC. Each of the PHY mRNAs fluctuate in a circadian fashion, but only abundance of PHYA, PHYB, and PHYC proteins oscillate, which may account for the varying response to red light throughout the circadian cycle. The $P R R 9$ gene is rapidly and transiently induced by white or red light in a phytochrome-dependent manner; PRR9 interacts with TOC1 through their pseudoreceiver domains, which provides additional evidence for the involvement of the PRR family in light signal transduction pathways. Blue light is transduced through PHYA as well as the CRYPTOCHROME (CRY1 and CRY2) 


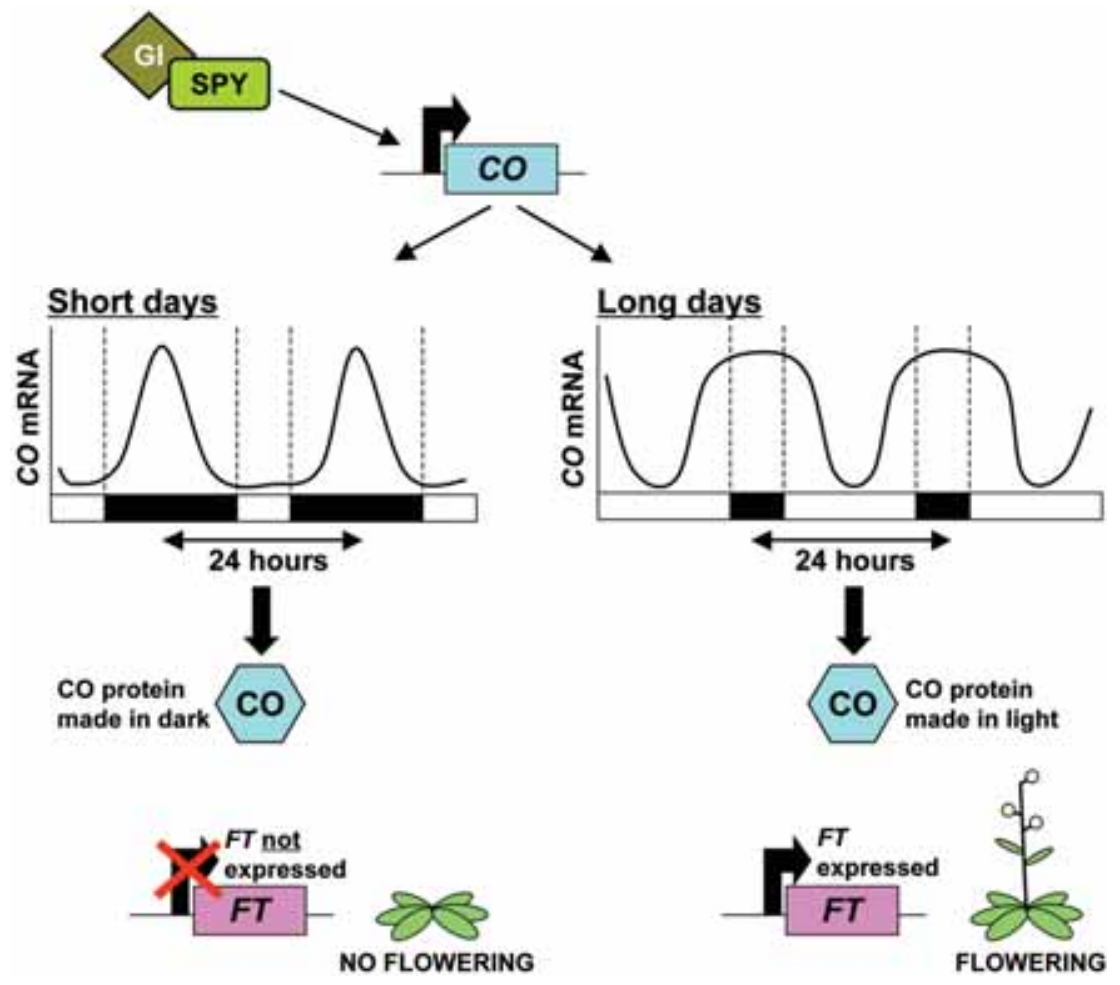

Figure 12. Circadian regulation of flowering in A. thaliana. During the short days of winter, the peak of $C O \mathrm{mRNA}$ and subsequent $\mathrm{CO}$ protein occurs during the night and does not promote the expression of the $F T$ gene; no flowering occurs. During the long days of summer, the pattern of $C O$ expression changes such that the peak of expression is more broad. This alteration results in high $C O$ expression in the early and late day. CO protein, which is activated by light, is active during these long days and activates expression of $F T$, which leads to flowering. (Figure and legend modified, with permission, from Putterill et al. 2004 [@ Wiley-Liss, a subsidiary of John Wiley \& Sons].)

proteins. ELF3 and TIME FOR COFFEE (TIC) abrogate light input to the clock. Specifically, ELF3 gates light input at dusk to allow for increased sensitivity by the clock to light at dawn.

Output. Temporal information from the oscillator controls biochemical/physiological pathways that regulate photosynthesis, cotyledon and leaf movement, rate of hypocotyl elongation, stomatal movement, and rhythmic accumulation of mRNA and protein that are involved in these processes. Although molecular output pathways in most organisms are not clearly understood, circadian regulation of the flowering pathway in Arabidopsis has been defined (Fig. 12) (for review, see Putterill et al. 2004). GI and SPINDLY (SPY) interact to activate circadian-regulated expression of CONSTANS (CO). In short days, a narrow peak of $C O$ occurs during the night; in long days, the peak of $C O$ becomes more broad to allow the peak to extend within the light period at dawn and in late afternoon. Because $\mathrm{CO}$ protein is activated by light, this coincidence of peak expression during the light phase produces sufficient active CO to induce FLOWERING LOCUS T (FT), which interacts with the bZIP transcription factor FD to activate expression of genes necessary for the transition from vegetative growth to flowering.

The enhanced fitness of plants that maintain internal time has been demonstrated in a number of different experiments. Arabidopsis plants with an endogenous circadian period that closely matches that of a given light/dark cycle produce more chlorophyll and fix more carbon (i.e., possess a photosynthetic advantage) than mutant plant lines that generate a period that is shorter or longer than the environmental cycle (Dodd et al. 2005). Plant lines that exist in different geographic regions are subjected to different light/dark cycles. The natural variation in the circadian timing of the plants correlates with the day length at the respective latitudes (Michael et al. 2003). This correlation suggests the evolutionary importance of tightly regulating internal timing to match the surrounding environment. Additional evidence for the adaptive advantage of maintaining an endogenous timekeeper is seen in plants that overexpress the core clock component CCA1 or LHY; these plants no longer regulate transcription in an anticipatory manner of light-todark transitions (Green et al. 2002). Consequently, these plants flower under long-day conditions later than their wild-type counterparts and are less viable under short-day conditions.

\section{ACKNOWLEDGMENTS}

I thank D. Bell-Pedersen, V.M. Cassone, S.S. Golden, P.E. Hardin, C.R. McClung, M.W. Vitalini, and M. Zatz for careful reading of sections of this manuscript. 


\section{REFERENCES}

Bell-Pedersen D., Cassone V.M., Earnest D.J., Golden S.S., Hardin P.E., Thomas T.L., and Zoran M.J. 2005. Circadian rhythms from multiple oscillators: Lessons from diverse organisms. Nat. Rev. Genet. 6: 544.

Ceriani M.F., Darlington T.K., Staknis D., Mas P., Petti A.A., Weitz C.J., and Kay S.A. 1999. Light-dependent sequestration of TIMELESS by CRYPTOCHROME. Science 285: 553.

Chen T.-H., Chen T.-L., Hung L.-M., and Huang T.-C. 1991. Circadian rhythm in amino acid uptake by Synechococcus RF-1. Plant Physiol. 97: 55.

Ditty J.L., Canales S.R., Anderson B.E., Williams S.B., and Golden S.S. 2005. Stability of the Synechococcus elongatus PCC 7942 circadian clock under directed anti-phase expression of the kai genes. Microbiology 151: 2605.

Dodd A.N., Salathia N., Hall A., Kevei E., Toth R., Nagy F., Hibberd J.M., Millar A.J., and Webb A.A. 2005. Plant circadian clocks increase photosynthesis, growth, survival, and competitive advantage. Science 309: 630

Doi M., Hirayama J., and Sassone-Corsi P. 2006. Circadian regulator CLOCK is a histone acetyltransferase. Cell 125: 497.

Dunlap J.C. and Loros J.J. 2006. How fungi keep time: Circadian system in Neurospora and other fungi. Curr. Opin. Microbiol. 9: 579.

Emery P., Stanewsky R., Hall J.C., and Rosbash M. 2000. A unique circadian-rhythm photoreceptor. Nature 404: 456.

Gachon F., Nagoshi E., Brown S.A., Ripperger J., and Schibler U. 2004. The mammalian circadian timing system: From gene expression to physiology. Chromosoma 113: 103.

Gardner M.J., Hubbard K.E., Hotta C.T., Dodd A.N., and Webb A.A. 2006. How plants tell the time. Biochem. J. 397: 15.

Golden S.S. and Canales S.R. 2003. Cyanobacterial circadian rhythms - Timing is everything. Nat. Rev. Microbiol. 1: 191.

Green R.M., Tingay S., Wang Z.Y., and Tobin E.M. 2002. Circadian rhythms confer a higher level of fitness to Arabidopsis plants. Plant Physiol. 129: 576.

Hardin P.E. 2006. Essential and expendable features of the circadian timekeeping mechanism. Curr. Opin. Neurobiol. 16: 686.

Hardin P.E. and Yu W. 2006. Circadian transcription: Passing the HAT to CLOCK. Cell 125: 424.

Herrero A., Muro-Pastor A.M., and Flores E. 2001. Nitrogen control in cyanobacteria. J. Bacteriol. 183: 411.

Ishiura M., Kutsuna S., Aoki S., Iwasaki H., Andersson C.R., Tanabe A., Golden S.S., Johnson C.H., and Kondo T. 1998. Expression of a gene cluster kaiABC as a circadian feedback process in cyanobacteria. Science 281: 1519.

Ivleva N.B., Bramlett M.R., Lindahl P.A., and Golden S.S. 2005. LdpA: A component of the circadian clock senses redox state of the cell. EMBO J. 24: 1202.

Ivleva N.B., Gao T., LiWang A.C., and Golden S.S. 2006. Quinone sensing by the circadian input kinase of the cyanobacterial circadian clock. Proc. Natl. Acad. Sci. 103: 17468.

Iwasaki H. and Kondo T. 2004. Circadian timing mechanism in the prokaryotic clock system of cyanobacteria. J. Biol. Rhythms 19: 436.

Iwasaki H., Nishiwaki T., Kitayama Y., Nakajima M., and Kondo T. 2002. KaiA-stimulated KaiC phosphorylation in circadian timing loops in cyanobacteria. Proc. Natl. Acad. Sci. 99: 15788 .

Johnson C.H. 2004. Global orchestration of gene expression by the biological clock of cyanobacteria. Genome Biol. 5: 217.

Katayama M., Kondo T., Xiong J., and Golden S.S. 2003. ldpA encodes an iron-sulfur protein involved in light-dependent modulation of the circadian period in the cyanobacterium Synechococcus elongatus PCC 7942. J. Bacteriol. 185: 1415.

Ko C.H. and Takahashi J.S. 2006. Molecular components of the mammalian circadian clock. Hum. Mol. Genet. (spec. no. 2) 15: R271.

Koh K., Zheng X., and Sehgal A. 2006. JETLAG resets the Drosophila circadian clock by promoting light-induced degradation of TIMELESS. Science 312: 1809.

Kondo T., Mori T., Lebedeva N.V., Aoki S., Ishiura M., and Golden S.S. 1997. Circadian rhythms in rapidly dividing cyanobacteria. Science 275: 224.

Kuhlman S.J. and McMahon D.G. 2006. Encoding the ins and outs of circadian pacemaking. J. Biol. Rhythms 21: 470.

Kutsuna S., Kondo T., Aoki S., and Ishiura M. 1998. A periodextender gene, pex, that extends the period of the circadian clock in the cyanobacterium Synechococcus sp. strain PCC 7942. J. Bacteriol. 180: 2167.

Kwon I., Lee J., Chang S.H., Jung N.C., Lee B.J., Son G.H., Kim K., and Lee K.H. 2006. BMAL1 shuttling controls transactivation and degradation of the CLOCK/BMAL1 heterodimer. Mol. Cell. Biol. 26: 7318.

Liu Y. and Bell-Pedersen D. 2006. Circadian rhythms in Neurospora crassa and other filamentous fungi. Eukaryot. Cell 5: 1184 .

Liu Y., Tsinoremas N.F., Johnson C.H., Lebedeva N.V., Golden S.S., Ishiura M., and Kondo T. 1995. Circadian orchestration of gene expression in cyanobacteria. Genes Dev. 9: 1469.

Mackey S.R. and Golden S.S. 2007. Winding up the cyanobacterial circadian clock. Trends Microbiol. 15: 381.

McClung C.R. 2006. Plant circadian rhythms. Plant Cell 18: 792.

Michael T.P., Salome P.A., Yu H.J., Spencer T.R., Sharp E.L., McPeek M.A., Alonso J.M., Ecker J.R., and McClung C.R. 2003. Enhanced fitness conferred by naturally occurring variation in the circadian clock. Science 302: 1049.

Mihalcescu I., Hsing W., and Leibler S. 2004. Resilient circadian oscillator revealed in individual cyanobacteria. Nature 430: 81 .

Mitsui A., Kumazawa S., Takahashi A., Ikemoto H., and Arai T. 1986. Strategy by which nitrogen-fixing unicellular cyanobacteria grow photoautotrophically. Nature 323: 720.

Mizuno T. and Nakamichi N. 2005. Pseudo-response regulators (PRRs) or true oscillator components (TOCs). Plant Cell Physiol. 46: 677.

Mori T. and Johnson C.H. 2000. Circadian control of cell division in unicellular organisms. Prog. Cell Cycle Res. 4: 185.

Mori T., Binder B., and Johnson C.H. 1996. Circadian gating of cell division in cyanobacteria growing with average doubling times of less than 24 hours. Proc. Natl. Acad. Sci. 93: 10183.

Nakajima M., Imai K., Ito H., Nishiwaki T., Murayama Y., Iwasaki H., Oyama T., and Kondo T. 2005. Reconstitution of circadian oscillation of cyanobacterial KaiC phosphorylation in vitro. Science 308: 414.

Ouyang Y., Andersson C.R., Kondo T., Golden S.S., and Johnson C.H. 1998. Resonating circadian clocks enhance fitness in cyanobacteria. Proc. Natl. Acad. Sci. 95: 8660.

Pattanayek R., Wang J., Mori T., Xu Y., Johnson C.H., and Egli M. 2004. Visualizing a circadian clock protein: Crystal structure of KaiC and functional insights. Mol. Cell 15: 375.

Putterill J., Laurie R., and Macknight R. 2004. It's time to flower: The genetic control of flowering time. Bioessays 26: 363.

Schmitz O., Katayama M., Williams S.B., Kondo T., and Golden S.S. 2000. CikA, a bacteriophytochrome that resets the cyanobacterial circadian clock. Science 289: 765.

Smith R.M. and Williams S.B. 2006. Circadian rhythms in gene transcription imparted by chromosome compaction in the cyanobacterium Synechococcus elongatus. Proc. Natl. Acad. Sci. 103: 8564

Sweeney B.M. and Borgese M.B. 1989. A circadian rhythm in cell division in a prokaryote, the cyanobacterium Synechococcus WH7803. J. Phycol. 25: 183.

Taghert P.H. and Shafer O.T. 2006. Mechanisms of clock output in the Drosophila circadian pacemaker system. J. Biol. Rhythms 21: 445.

Takai N., Ikeuchi S., Manabe K., and Kutsuna S. 2006a. Expression of the circadian clock-related gene pex in cyanobacteria increases in darkness and is required to delay the clock. J. Biol. Rhythms 21: 235.

Takai N., Nakajima M., Oyama T., Kito R., Sugita C., Sugita M., Kondo T., and Iwasaki H. 2006b. A KaiC-associating SasARpaA two-component regulatory system as a major circadian 


\section{BIOLOGICAL RHYTHMS WORKSHOP IA}

timing mediator in cyanobacteria. Proc. Natl. Acad. Sci. 103: 12109.

Taniguchi Y., Katayama M., Ito R., Takai N., Kondo T., and Oyama T. 2007. labA: A novel gene required for negative feedback regulation of the cyanobacterial circadian clock protein KaiC. Genes Dev. 21: 60.

Vitalini M.W., de Paula R.M., Park W.D., and Bell-Pedersen D. 2006. The rhythms of life: Circadian output pathways in Neurospora. J. Biol. Rhythms 21: 432.

Williams S.B. 2006. A circadian timing mechanism in the cyanobacteria. Adv. Microb. Physiol. 52: 229.

Woelfle M.A. and Johnson C.H. 2006. No promoter left behind:
Global circadian gene expression in cyanobacteria. J. Biol. Rhythms 21: 419

Xu Y., Mori T., and Johnson C.H. 2000. Circadian clock-protein expression in cyanobacteria: Rhythms and phase setting. EMBO J. 19: 3349

- 2003. Cyanobacterial circadian clockwork: Roles of $\mathrm{KaiA}, \mathrm{KaiB}$ and the $\mathrm{kaiBC}$ promoter in regulating KaiC. EMBO J. 22: 2117.

Xu Y., Mori T., Pattanayek R., Pattanayek S., Egli M., and Johnson C.H. 2004. Identification of key phosphorylation sites in the circadian clock protein KaiC by crystallographic and mutagenetic analyses. Proc. Natl. Acad. Sci. 101: 13933. 


\section{$8_{\mathrm{CSH}}^{\infty} \mathrm{CH}$ Cold Spring Harbor Symposia SYMPOSIA on Quantitative Biology}

\section{Biological Rhythms Workshop IA: Molecular Basis of Rhythms Generation}

S. R. Mackey

Cold Spring Harb Symp Quant Biol 2007 72: 7-19

Access the most recent version at doi:10.1101/sqb.2007.72.060

References This article cites 55 articles, 28 of which can be accessed free at:

http://symposium.cshlp.org/content/72/7.full.html\#ref-list-1

License

Email Alerting Receive free email alerts when new articles cite this article - sign up in the box at the Service top right corner of the article or click here.

To subscribe to Cold Spring Harbor Symposia on Quantitative Biology go to: http://symposium.cshlp.org/subscriptions 